\title{
RADIATIVELY INEFFICIENT ACCRETION DISKS
}

\author{
H.C. SPRUIT \\ Max-Planck-Institut für Astrophysik \\ Postfach 1523, D-85740 Garching, Germany
}

\begin{abstract}
Radiatively inefficient (or advection dominated) disks are discussed at an introductory level. Ion supported and radiation supported flows are discussed, the different consequences of advection dominated flows onto black holes vs. solid surfaces (neutron stars, white dwarfs), hydrodynamics, the role of the ratio of specific heats, and the possible connection between ADAFs and outflows.
\end{abstract}

Key words: neutron stars, black holes, accretion: accretion disks

\section{Introduction}

In a thin accretion disk, the time available for the accreting gas to radiate away the energy released by the viscous stress is the accretion time,

$$
t_{\mathrm{acc}} \approx \frac{1}{\alpha \Omega_{\mathrm{K}}}\left(\frac{r}{H}\right)^{2},
$$

where $\alpha$ is the dimensionless viscosity parameter, $\Omega_{\mathrm{K}}$ the local Keplerian rotation rate, $r$ the distance from the central mass, and $H$ the disk thickness (see Frank et al. 1992 or Spruit, elsewhere in this volume). For a thin disk, $H / r \ll 1$, this time is much longer than the thermal time scale $t_{\mathrm{t}} \approx 1 /(\alpha \Omega)$. There is then enough time for a local balance to exist between viscous dissipation and radiative cooling. For the accretion rates implied in observed systems the disk is then rather cool, and the starting assumption $H / r \ll 1$ is justified.

This argument is somewhat circular, however, since the accretion time is long enough for effective cooling only if the disk is assumed to be thin to begin with. Other forms of accretion disks may exist, even at the same 
accretion rates, in which the cooling is ineffective compared with that of standard (geometrically thin, optically thick) disks.

Since radiatively inefficient disks tend to be thick, $H / r \sim O(1)$, they are sometimes called 'quasi-spherical'. However, this does not mean that a spherically symmetric accretion model would be a reasonable approximation. The crucial difference is that the flow has angular momentum. The inward flow speed is governed by the rate at which angular momentum can be transferred outwards, rather than by gravity and pressure gradient. The accretion time scale, $t_{\mathrm{acc}} \sim 1 /(\alpha \Omega)$ is longer than the accretion time scale in the spherical case (unless the viscosity parameter $\alpha$ is as large as $O(1)$ ). The dominant velocity component is azimuthal rather than radial, and the density and optical depth are much larger than in the spherical case.

It turns out that there are two kinds of radiatively inefficient disks, the optically thin, and optically thick varieties. A second distinction occurs because accretion flows are different for central objects with a solid surface (neutron stars, white dwarfs, main sequence stars, planets), and those without (i.e. black holes). I start with optically thick flows.

\section{Radiation supported advective accretion}

If the energy loss by radiation is small, the gravitational energy release $W_{\text {grav }} \approx G M /(2 r)$ is converted into enthalpy of the gas and radiation field ${ }^{1}$

$$
\frac{1}{2} \frac{G M}{r}=\frac{1}{\rho}\left[\frac{\gamma}{\gamma-1} P_{\mathrm{g}}+4 P_{\mathrm{r}}\right]
$$

where an ideal gas of constant ratio of specific heats $\gamma$ has been assumed, and $P_{\mathrm{r}}=\frac{1}{3} a T^{4}$ is the radiation pressure. In terms of the virial temperature $T_{\text {vir }}=G M /(\mathcal{R} r)$, and assuming $\gamma=5 / 3$, appropriate for a fully ionized gas, this can be written as

$$
\frac{T}{T_{\mathrm{vir}}}=\left[5+8 \frac{P_{\mathrm{r}}}{P_{\mathrm{g}}}\right]^{-1}
$$

Thus, for radiation pressure dominated accretion, $P_{\mathrm{r}} \gg P_{\mathrm{g}}$, the temperature is much less than the virial temperature. The disk thickness is given by

$$
H \approx\left[\left(P_{\mathrm{g}}+P_{\mathrm{r}}\right) / \rho\right]^{1 / 2} / \Omega
$$

With (3) this yields

$$
H / r \sim O(1)
$$

\footnotetext{
${ }^{1}$ I assume here that a fraction $\sim 0.5$ of the gravitational potential energy stays in the flow as orbital kinetic energy. See also section 3.
} 
In the limit $P_{\mathrm{r}} \gg P_{\mathrm{g}}$, the flow is therefore geometrically thick. This implies that radiation pressure supplies a non-negligible fraction of the support of the gas against gravity (the remainder being provided by rotation).

For $P_{\mathrm{r}} \gg P_{\mathrm{g}},(2)$ yields

$$
\frac{G M}{2 r}=\frac{4}{3} \frac{a T^{4}}{\rho} .
$$

The radiative energy flux, in the diffusion approximation, is

$$
F=\frac{4}{3} \frac{\mathrm{d}}{\mathrm{d} \tau} \sigma T^{4} \approx \frac{4}{3} \frac{\sigma T^{4}}{\tau},
$$

where $\sigma=a c / 4$ is Stefan-Boltzmann's radiation constant. Hence

$$
F=\frac{1}{8} \frac{G M}{r H} \frac{c}{\kappa}=F_{\mathrm{E}} \frac{r}{8 H},
$$

where $F_{\mathrm{E}}=L_{\mathrm{E}} /\left(4 \pi r^{2}\right)$ is the local Eddington flux. Since $H / r \approx 1$, a radiatively inefficient, radiation pressure dominated accretion flow has a luminosity of the order of the Eddington luminosity.

The temperature depends on the accretion rate and the viscosity $\nu$ assumed. The accretion rate is of the order $\dot{M} \sim 3 \pi \nu \Sigma$ (see 'accretion disks' elsewhere in this volume), where $\Sigma=\int \rho \mathrm{d} z$ is the surface mass density. In units of the Eddington rate, we get

$$
\dot{m} \equiv \dot{M} / \dot{M}_{\mathrm{E}} \approx \nu \rho \kappa / c,
$$

where $H / r \approx 1$ has been used, and $\dot{M}_{\mathrm{E}}$ is the Eddington accretion rate onto the central object of size $R$,

$$
\dot{M}_{\mathrm{E}}=\frac{R}{G M} L_{\mathrm{E}}=4 \pi R c / \kappa .
$$

[Note that the definition of $\dot{M}_{\mathrm{E}}$ differs by factors of order unity between different authors. It depends on the assumed efficiency $\eta$ of conversion of gravitational energy $G M / R$ into radiation. In (10) it is taken to be unity, for accretion onto black holes a more realistic value is $\eta=0.1$, for accretion onto neutron stars $\eta \approx 0.4$.]

Assume that the viscosity scales with the gas pressure:

$$
\nu=\alpha \frac{P_{\mathrm{g}}}{\rho \Omega_{\mathrm{K}}}
$$

instead of the total pressure $P_{\mathrm{r}}+P_{\mathrm{g}}$. This is the form that is likely to hold if the angular momentum transport is due to a small-scale magnetic 
field (Sakimoto and Coroniti, 1989). Then with (6) we have (up to a factor $2 H / r \sim O(1)$

$$
T^{5} \approx \frac{(G M)^{3 / 2}}{r^{5 / 2}} \frac{\dot{m} c}{\alpha \kappa a \mathcal{R}}
$$

or

$$
T \approx 210^{8} r_{6}^{-1 / 5}\left(r / r_{\mathrm{g}}\right)^{3 / 10} \dot{m}^{1 / 5},
$$

where $r=10^{6} r_{6}$ and $r_{\mathrm{g}}=2 \mathrm{GM} / \mathrm{c}^{2}$ is the gravitational radius of the accreting object, and the electron scattering opacity of $0.3 \mathrm{~cm}^{2} / \mathrm{g}$ has been assumed. The temperatures expected in radiation supported advection dominated flows are therefore quite low compared with the virial temperature [If the viscosity is assumed to scale with the total pressure instead of $P_{\mathrm{g}}$, the temperature is even lower]. The effect of electron-positron pairs can be neglected (Schultz and Price, 1985), since they are present only at temperatures approaching the electron rest mass energy, $T \gtrsim 10^{9} \mathrm{~K}$.

In order for the flow to be radiation pressure and advection dominated, the optical depth has to be sufficiently large so the radiation does not leak out. The energy density in the flow, vertically integrated at a distance $r$, is of the order

$$
E \approx a T^{4} H
$$

and the energy loss rate per $\mathrm{cm}^{2}$ of disk surface is given by (8). The cooling time is therefore,

$$
t_{\mathrm{c}}=E / F=3 \tau H / c .
$$

This is to be compared with the accretion time, which can be written in terms of the mass in the disk at radius $r$, of the order $2 \pi r^{2} \Sigma$, and the accretion rate:

$$
t_{\mathrm{acc}}=2 \pi r^{2} \Sigma / \dot{M}
$$

This yields

$$
t_{\mathrm{c}} / t_{\mathrm{acc}} \approx \frac{\kappa}{\pi r c} \dot{M}=4 \dot{m} \frac{R}{r},
$$

(where a factor $3 / 2 H / r \sim O(1)$ has been neglected). Since $r>R$, this shows that accretion has to be super-Eddington in order to be both radiationand advection-dominated.

This condition can also be expressed in terms of the so-called trapping radius $r_{\mathrm{t}}$ (e.g. Rees 1978). Equating $t_{\mathrm{acc}}$ and $t_{\mathrm{c}}$ yields

$$
r_{\mathrm{t}} / R \approx 4 \dot{m}
$$

Inside $r_{\mathrm{t}}$, the flow is advection dominated: the radiation field produced by viscous dissipation stays trapped inside the flow, instead of being radiated from the disk as happens in a standard thin disk. Outside the trapping 
radius, the radiation field can not be sufficiently strong to maintain a disk with $H / r \sim 1$, it must be a thin form of disk instead. Such a thin disk can still be radiation-supported (i.e. $P_{\mathrm{r}} \gg P_{\mathrm{g}}$ ), but it can not be advection dominated.

Flows of this kind are called 'radiation supported tori' (or radiation tori, for short) by Rees et al. $1982^{2}$. They must accrete at a rate above the Eddington value to exist. The converse is not quite true: a flow accreting above Eddington is an advection dominated flow, but it need not necessarily be radiation dominated. Advection dominated optically thick acretion flows exist in which radiation does not play a major role (see section 3.1).

That an accretion flow above $\dot{M}_{\mathrm{E}}$ is advection dominated, not a thin disk, follows from the fact that in a thin disk the energy dissipated must be radiated away locally. Since the local radiative flux can not exceed the Eddington energy flux $F_{\mathrm{E}}$, the mass accretion rate in a thin disk can not significantly exceed the Eddington value (10).

The gravitational energy, dissipated by viscous stress in differential rotation and advected with the flow, ends up at the central object. If this is a black hole, the photons, particles and their thermal energy are conveniently swallowed at the horizon, and do not react back on the flow. Radiation tori are therefore mostly relevant for accretion onto black holes. They are convectively unstable (Bisnovatyi-Kogan and Blinnikov 1977): the way in which energy is dissipated, in the standard $\alpha$-prescription, is such that the entropy $\left(\sim T^{3} / \rho\right)$ decreases with height in the disk. Recent numerical simulations (see section 5 ) show the effects of this convection.

\subsection{SUPER-EDDINGTON ACCRETION ONTO BLACK HOLES}

As the accretion rate onto a black hole is increased above $\dot{M}_{\mathrm{E}}$, the trapping radius moves out. The total luminosity increases only slowly, and remains of the order of the Eddington luminosity. Such supercritical accretion has been considered by Begelman and Meier (1982, see also Wang and Zhou 1999); they show that the flow has a radially self-similar structure.

Abramowicz et al. $(1988,1989)$ studied accretion onto black holes at rates near $\dot{M}_{\mathrm{E}}$. They used a vertically-integrated approximation for the disk, but included the advection terms. The resulting solutions were called 'slim disks'. These models show how with increasing accretion rate, a standard thin Shakura-Sunyaev disk turns into a radiation-supported advection flow. The nature of the transition depends on the viscosity prescription

\footnotetext{
${ }^{2}$ Some workers interpret the use of word 'torus' in the context radiatively inefficient accretion as implying a rotating but non-accreting flow. The physics studied by Rees et al. (1982), however, where this name was introduced, explicitly refers to accreting flows such as described here
} 
used, and can show a non-monotonic dependence of $\dot{M}$ on surface density $\Sigma$ (Honma et al. 1991). This suggests the possibility of instability and cyclic behavior of the inner disk near a black hole, at accretion rates near and above $\dot{M}_{\mathrm{E}}$ (for an application to GRS 1915+105 see Nayakshin et al., 1999).

\subsection{SUPER-EDDINGTON ACCRETION ONTO NEUTRON STARS}

In the case of accretion onto a neutron star, the energy trapped in the flow, plus the remaining orbital energy, settles onto its surface. If the accretion rate is below $\dot{M}_{\mathrm{E}}$, the energy can be radiated away by the surface, and steady accretion is possible. A secondary star providing the mass may, under some circumstances, transfer more than $\dot{M}_{\mathrm{E}}$, since it does not know about the neutron star's Eddington value. The outcome of this case is still somewhat uncertain; it is generally believed on intuitive grounds that the 'surplus' (the amount above $\dot{M}_{\mathrm{E}}$ ) somehow gets expelled from the system.

As the transfer rate is increased, the accreting hot gas forms an extended atmosphere around the neutron star, like the envelope of a giant. If it is large enough, the outer parts of this envelope are partially ionized. The opacity in these layers, due to lines of the CNO and heavier elements, is then much higher than the electron scattering opacity. The Eddington luminosity based on the local value of the opacity is then smaller than it is near the neutron star surface. Once an extended atmosphere with a cool surface forms, the the accretion luminosity is thus large enough to drive a wind from the envelope (see Kato 1997, where this is discussed in the context of Novae).

This scenario is somewhat dubious however, since it assumes that the mass transferred from the secondary continues to reach the neutron star and generate a high luminosity there. This is not at all obvious, since the mass transfering stream may dissipate in the growing neutron star envelope instead. The result would be a giant (more precisely, a Thorne-Zytkow star), with a steadily increasing envelope mass. Such an envelope is likely to be large enough to envelop the entire binary system, which then develops into a common-envelope (CE) system. The envelope mass is expected to be ejected by CE hydrodynamics (Taam 1994, 2000).

A more speculative proposal, suggested by the properties of SS 433, is that the 'surplus mass' is ejected in the form of jets. The binary parameters of Cyg X-2 are observational evidence for mass ejection in super-Eddington mass transfer phases (King and Ritter 1999, Rappaport and Podsiadlowski 1999, King and Begelman 1999). 


\section{Hydrodynamics}

The hydrodynamics of ADAFs and radiation tori can be studied by starting, at a very simple level, with a generalization of the thin disk equations. Making the assumption that quantities integrated over the height $z$ of the disk give a fair representation (though this is justifiable only for thin disks), and assuming axisymmetry, the problem reduces to a one-dimensional timedependent one. Further simplifying this by restriction to a steady flow yields the equations

$$
\begin{gathered}
2 \pi r \Sigma v_{r}=\dot{M}=\mathrm{cst}, \\
r \Sigma v_{r} \partial_{r}\left(\Omega r^{2}\right)=\partial_{r}\left(\nu \Sigma r^{3} \partial_{r} \Omega\right), \\
v_{r} \partial_{r} v_{r}-\left(\Omega^{2}-\Omega_{\mathrm{K}}^{2}\right) r=-\frac{1}{\rho} \partial_{r} p, \\
\Sigma v_{r} T \partial_{r} S=q^{+}-q^{-},
\end{gathered}
$$

where $S$ is the specific entropy of the gas, $\Omega$ the local rotation rate, now different from the Keplerian rate $\Omega_{\mathrm{K}}=\left(G M / r^{3}\right)^{1 / 2}$, while

$$
q^{+}=\int Q_{\mathrm{v}} \mathrm{d} z \quad q^{-}=\int \operatorname{div} F_{\mathrm{r}} \mathrm{d} z
$$

are the height-integrated viscous dissipation rate and radiative loss rate, respectively. In the case of thin disks, equations (19) and (20) are unchanged, but (21) simplifies to $\Omega^{2}=\Omega_{\mathrm{K}}^{2}$, i.e. the rotation is Keplerian, while (22) simplifies to $q^{+}=q^{-}$, expressing local balance between viscous dissipation and cooling. The left hand side of (22) describes the radial advection of heat, and is perhaps the most important deviation from the thin disk equations at this level of approximation (hence the name advection dominated

flows). The characteristic properties are seen most cearly when radiative loss is neglected altogether, $q^{-}=0$. The equations are supplemented with expressions for $\nu$ and $q^{+}$:

$$
\nu=\alpha c_{\mathrm{s}}^{2} / \Omega_{\mathrm{K}} ; \quad q^{+}=\left(r \partial_{r} \Omega\right)^{2} \nu \Sigma .
$$

If $\alpha$ is taken constant, $q^{-}=0$, and an ideal gas is assumed with constant ratio of specific heats, so that the entropy is given by

$$
S=c_{\mathrm{v}} \ln \left(p / \rho^{\gamma}\right),
$$

then equations (19)-(22) have no explicit length scale in them. This means that a special so-called self-similar solution exists, in which all quantities are powers of $r$. Such self-similar solutions have apparently first been described 
by Gilham (1981), but has since then been re-invented several times (Spruit et al. 1987; Narayan and Yi, 1994). The dependences on $r$ are

$$
\begin{aligned}
\Omega \sim r^{-3 / 2} ; & \rho & \sim r^{-3 / 2}, \\
H \sim r ; & T & \sim r^{-1} .
\end{aligned}
$$

In the limit $\alpha \ll 1$, one finds

$$
\begin{gathered}
v_{r}=-\alpha \Omega_{\mathrm{K}} r\left(9 \frac{\gamma-1}{5-\gamma}\right), \\
\Omega=\Omega_{\mathrm{K}}\left(2 \frac{5-3 \gamma}{5-\gamma}\right)^{1 / 2}, \\
c_{\mathrm{s}}^{2}=\Omega_{\mathrm{K}}^{2} r^{2} \frac{\gamma-1}{5-\gamma} \\
\frac{H}{r}=\left(\frac{\gamma-1}{5-\gamma}\right)^{1 / 2} .
\end{gathered}
$$

The precise from of these expressions depends somewhat on the way in which vertical integrations such as in (23) are done (which are only approximate).

The self-similar solution can be compared with numerical solutions of eqs. (19)-(22) with appropriate conditions applied at inner $\left(r_{\mathrm{i}}\right)$ and outer $\left(r_{\mathrm{o}}\right)$ boundaries (Nakamura et al. 1996, Narayan et al. 1997). The results show that the self-similar solution is valid in an intermediate regime $r_{\mathrm{i}} \ll$ $r \ll r_{\mathrm{o}}$. That is, the solutions of (19)-(22) approach the self-similar solution far from the boundaries, as is characteristic of self-similar solutions.

The solution exists only if $1<\gamma \leq 5 / 3$, a condition satisfied by all ideal gases. As $\gamma \downarrow 1$, the disk temperature and thickness vanish. This is understandable, since a $\gamma$ close to 1 means that the gas has a large number of internal degrees of freedom. The accretion energy is shared between all degrees of freedom, so that for a low $\gamma$ less is available for the kinetic energy (temperature) of the particles.

Second, the rotation rate vanishes for $\gamma \rightarrow 5 / 3$. Since a fully ionized gas has $\gamma=5 / 3$, it is the most relevant value for optically thin accretion near a black hole or neutron star. Apparently, steady advection dominated accretion can not have angular momentum in this case, and the question arises how an adiabatic flow with angular momentum will behave for $\gamma=5 / 3$. In the literature, this problem has been circumvented by arguing that real flows would have magnetic fields in them, which would change the effective compressibility of the gas. Even if a magnetic field of sufficient strength is present, however, (energy density comparable to the gas pressure) the 
effective $\gamma$ is not automatically lowered. If the field is compressed mainly perpendicular to the field lines, for example, the effective $\gamma$ is closer to 2 . Also, this does not solve the conceptual problem what would happen to a rotating accretion flow consisting of a more weakly magnetized ionized gas.

This conceptual problem has been solved by Ogilvie (1999), who showed how the low rotation for $\gamma=5 / 3$ comes about in a time-dependent manner. He found a similarity solution (depending on distance and time in the combination $r / t^{2 / 3}$ ) to the time-dependent version of eqs (19)-(22). This solution describes the asymptotic behavior (in time) of a viscously spreading disk, analogous to the viscous spreading of thin disks (Lynden-Bell and Pringle 1972). As in the thin disk case, all the mass accretes asymptotically onto the central mass, while all the angular momentum travels to infinity together with a vanishing amount of mass. For all $\gamma<5 / 3$, the rotation rate at a fixed $r$ tends to a finite value as $t \rightarrow \infty$, but for $\gamma=5 / 3$ it tends to zero. The slowly-rotating region expands in size as $r \sim t^{2 / 3}$. It thus seems likely that the typical slow rotation of ADAFs at $\gamma$ near $5 / 3$ is a real physical property, and that the angular momentum gets expelled from the inner regions of the flow.

\subsection{OTHER OPTICALLY THICK ACCRETION FLOWS}

The radiation-dominated flows discussed in section 2 are not the only possible optically thick advection dominated flows. From the discussion of the hydrodynamics, it is clear that disk-like (i.e. rotating) accretion is possible whenever the ratio of specific heats is less than 5/3. A radiation supported flow satisfies this requirement with $\gamma=4 / 3$, but it can also happen in the absence of radiation if energy is taken up in the gas by internal degrees of freedom of the particles. Examples are the rotational and vibrational degrees of freedom in molecules, and the energy associated with dissociation and ionization. If the accreting object has a gravitational potential not much exceeding the $13.6+2.2 \mathrm{eV}$ per proton for dissociation plus ionization, a gas initially consisting of molecular hydrogen can stay bound at arbitrary accretion rates. This translates into a limit $M / M_{\odot} R_{\odot} / R<0.01$. This is satisfied approximately by the giant planets, which are believed to have gone through a phase of rapid adiabatic gas accretion (e.g. Podolak et al. 1993).

A more remotely related example is the core-collapse supernova. The accretion energy of the envelope mass falling onto the proto-neutron star is lost mostly through photodisintegration of nuclei, causing the well known problem of explaining how a shock is produced of sufficient energy to unbind the envelope. If the pre-collapse core rotates sufficiently rapidly, the collapse will form an accretion torus (inside the supernova envelope), with properties 
similar to advection dominated accretion flows (but at extreme densities and accretion rates, by X-ray binary standards). Such objects have been invoked as sources of Gamma-ray bursts (Popham et al. 1999, see also the review by Meszaros, elsewhere in this volume).

A final possibility for optically thick accretion is through neutrino losses. If the temperature and density near an accreting neutron star become large enough, additional cooling takes place through neutrinos (as in the cores of giants). This is relevant for the physics of Thorne-Zytkow stars (neutron stars or black holes in massive supergiant envelopes, cf. Bisnovatyi-Kogan and Lamzin 1984, Cannon et al. 1992), and perhaps for the spiral-in of neutron stars into giants (Chevalier 1993, see however Taam 2000).

\section{Optically thin advection dominated flows (ADAFs)}

The optically thin case has received most attention in recent years, because of the promise it holds for explaining the (radio to X-ray) spectra of X-ray binaries and the central black holes in galaxies, including our own. For a recent review see Yi (1999). This kind of flow occurs if the gas is optically thin, and radiation processes sufficiently weak. The gas then heats up to near the virial temperature. Near the last stable orbit of a black hole, this is of the order $100 \mathrm{MeV}$, or $10^{12} \mathrm{~K}$. At such temperatures, a gas in thermal equilibrium would radiate at a fantastic rate, even if it were optically thin, because the interaction between electrons and photons becomes very strong already near the electron rest mass of $0.5 \mathrm{MeV}$. In a remarkable paper, Shapiro Lightman and Eardley (1976) noted that this, however, is not what will happen in an optically thin accreting plasma but that, instead, a twotemperature plasma forms.

Suppose that the energy released by viscous dissipation is distributed equally among the carriers of mass, i.e. mostly to the ions and $\sim 1 / 2000$ to the electrons. Most of the energy resides in the ions, which radiate very inefficiently (their high mass prevents the rapid accelerations that are needed to produce electromagnetic radiation). This energy is transfered to the electrons by Coulomb interactions. These interactions are slow, however, under the conditions mentioned. They are slow because of the low density (on account of the assumed optical tickness), and because they decrease with increasing temperature. The electric forces that transfer energy from an ion to an electron act only as long as the ion is within the electron's Debye sphere (e.g. Spitzer, 1965). The interaction time between proton and electron, and

thus the momentum transfered, therefore decrease as $1 / v_{\mathrm{p}} \sim T_{\mathrm{p}}^{-1 / 2}$ where $T_{\mathrm{p}}$ is the proton temperature.

In this way, an optically thin plasma near a compact object can be in a two-temperature state, with the ions being near the virial temperature, and 
the electrons, which are doing the radiating, at a much lower temperature around 50-200 keV. The energy transfer from the gravitational field to the ions is fast (by some form of viscous or magnetic dissipation), from the ions to the electrons slow, and finally the energy losses of the electrons fast (by synchrotron radiation in a magnetic field or by inverse Compton scattering of soft photons). Such a flow would be radiatively inefficient since the receivers of the accretion energy, the ions, get swallowed by the hole before getting a chance to transfer their energy to the electrons. The first disk models which take into account the physics of advection and a two-temperature plasma were developed by Ichimaru (1977).

It is clear from this description that both the physics of such flows and the radiation spectrum to be expected depend crucially on the details of the ion-electron interaction and radiation processes assumed. This is unlike the case of the optically thick advection dominated flows, where gas and radiation are in approximate thermodynamic equilibrium. This is a source of uncertainty in the application of the optically thin ADAFs to observed systems, since their radiative properties depend on poorly known quantities, for example, the strength of the magnetic field in the flow.

The various branches of optically thin and thick accretion flows are summarized in figure 1. Each defines a relation between surface density $\Sigma$ (or optical depth $\tau=\kappa \Sigma$ ) and accretion rate. Optically thin ADAFs require low densities, either because of low accretion rates or large values of the viscosity parameter. The condition that the cooling time of the ions by energy transfer to the electrons is longer than the accretion time yields a maximum accretion rate (Rees et al. 1982),

$$
\dot{m} \lesssim \alpha^{2} .
$$

If $\alpha \approx 0.05$ as suggested by current simulations of magnetic turbulence, the maximum accretion rate would be a few $10^{-3}$. If ADAFs are to be applicable to systems with higher accretion rates, such as Cyg X-1 for example, the viscosity parameter must be larger, on the order of 0.3 .

\subsection{APPLICATION: HARD SPECTRA IN X-RAY BINARIES}

In the hard state, the X-ray spectrum of black hole and neutron star accreters is characterized by a peak in the energy distribution $\left(\nu F_{\nu}\right.$ or $\left.E F(E)\right)$ at photon energies around $100 \mathrm{keV}$. This is to be compared with the typical photon energy of $\sim 1 \mathrm{keV}$ expected from a standard optically thick thin disk accreting near the Eddington limit. The standard, and by far most likely explanation is that the observed hard photons are softer photons (around $1 \mathrm{keV}$ ) that have been up-scattered through inverse Compton scattering on hot electrons. Fits of such Comptonized spectra (e.g. Zdziarski 1998 and 


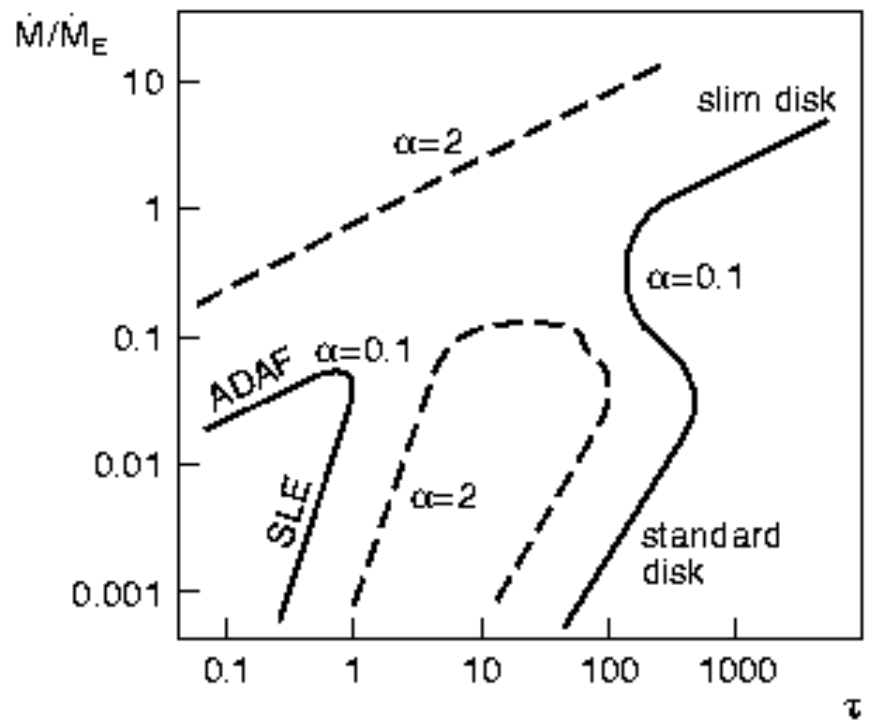

Figure 1. Branches of advection-dominated and thin disks for two values or the viscosity parameter $\alpha$, as functions of accretion rate and (vertical) optical depth of the flow (schematic, after Chen et al. 1995, Zdziarski 1998). Optically thin branches are the ADAF and SLE (Shapiro-Lightman-Eardley) solutions, optically thick ones the radiation dominated ('slim disk' or 'radiation torus') and SS (Shakura-Sunyaev or standard thin disk). Advection dominated are the ADAF and radiation torus, geometrically thin are the SLE and SS. The SLE solution is a thermally unstable branch.

references therein) yield an electron scattering optical depth around unity and an electron temperature of $50-100 \mathrm{keV}$. The scatter in these parameters is rather small between different sources. The reason may lie in part in the physics of Comptonization, but is not fully understood either. Something in the physics of the accretion flow keeps the Comptonization parameters constant as long as it is in the hard state. ADAFs have been applied with some success in interpreting XRB. They can produce reasonable X-ray spectra, and have been used in interpretations of the spectral-state transitions in sources like Cyg X-1 (Esin et al. 1998 and references therein).

An alternative to the ADAF model for the hard state in sources like Cyg $\mathrm{X}-1$ and the black hole X-ray transients is the 'corona' model. A hot corona (Bisnovatyi-Kogan and Blinnikov 1976), heated perhaps by magnetic fields as in the case of the Sun (Galeev et al. 1979) could be the medium that Comptonizes soft photons radiated from the cool disk underneath. The energy balance in such a model produces a Comptonized spectrum within the observed range (Haardt and Maraschi 1993). This model has received further momentum, especially as a model for AGN, with the discovery of 
broadened X-ray lines indicative of the presence of a cool disk close to the last stable orbit around a black hole (Lee et al. 1999 and references therein). The very rapid X-ray variability seen in some of these sources is interpreted as due magnetic flaring in the corona, like in the solar corona (e.g. Di Matteo et al. 1999a).

\subsection{TRANSITION FROM THIN DISK TO ADAF}

One of the difficulties in applying ADAFs to specific observed systems is the transition from a standard geometrically thin, optically thick disk, which must be the mode of mass transfer at large distances, to an ADAF at closer range. This is shown by figure 1, which illustrates the situation at some distance close to the central object. The standard disk and the optically thin branches are separated from each other for all values of the viscosity parameter. This separation of the optically thin solutions also holds at larger distances. Thus, there is no plausible continuous path from one to the other, and the transition between the two must be due to additional physics that is not included in diagrams like figure 1 .

A promising possibility is that the transition takes place through a gradual (as a function of radius) evaporation (Meyer and Meyer-Hofmeister 1994, Meyer-Hofmeister and Meyer 1999). In this scenario, evaporation initially produces a corona above the disk, which transforms into an ADAF further in.

\subsection{QUIESCENT GALACTIC NUCLEI}

For very low accretion rates, such as inferred for the black hole in the center of our galaxy (identified with the radio source Sgr $A^{*}$ ), the broad band spectral energy distribution of an ADAF is predicted to have two humps (Narayan et al. 1995, Quataert et al. 1999). In the X-ray range, the emission is due to bremsstrahlung. In the radio range, the flow emits synchrotron radiation, provided that the magnetic field in the flow has an energy density order of the gas pressure ('equipartition'). Synthetic ADAF spectra can be fitted to the observed radio and X-ray emission from Sgr $A^{*}$. In other galaxies where a massive central black hole is inferred, and the center is populated by an X-ray emitting gas of known density, ADAFs would also be natural, and might explain why the observed luminosities are so low compared with the accretion rate expected for a hole embedded in a gas of the measured density. In some of these galaxies, however, the peak in the radio-to-mm range predicted by analogy with Sgr A* is not observed (Di Matteo et al. 1999b). This requires an additional hypothesis, for example that the magnetic field in these cases is much lower, or that the accretion energy is carried away by an outflow. 


\subsection{TRANSIENTS IN QUIESCENCE}

$\mathrm{X}$-ray transients in quiescence (i.e. after an outburst) usually show a very low X-ray luminosity. The mass transfer rate from the secondary in quiescence can be inferred from the optical emission. This shows the characteristic 'hot spot', known from other systems to be the location where the mass transfering stream impacts on the edge of an accretion disk (e.g. Van Paradijs and McClintock 1995). These observations thus show that a disk is present in quiescence, while the mass transfer rate can be measured from the brightness of the hot spot. If this disk were to extend to the neutron star with constant mass flux, the predicted X-ray luminosity would be much higher than observed. This has traditionally been interpreted as a consequence of the fact that in transient systems, the accretion is not steady. Mass is stored in the outer parts and released by a disk instability (e.g. King 1995, Meyer-Hofmeister and Meyer 1999) producing the X-ray outburst. During quiescence, the accretion rate onto the compact object is much smaller than the mass transfer from the secondary to the disk.

ADAFs have been invoked as an alternative explanation. The quiescent accretion rate onto the central object is proposed to be higher than in the disk-instability explanation, the greater energy release being hidden on account of the low radiative efficiency of the ADAF. Some transient systems have neutron star primaries, with a hard surface at which the energy accreted by the ADAF must somehow be radiated away. A neutron star, with or without ADAFs, can not accrete in a radiatively inefficient way. In order to make ADAFs applicable, it has been proposed that the neutron stars in these systems have a modest magnetic dipole moment, such that in quiescence the gas in the accretion disk is prevented, by the 'propeller effect' (Illarionov and Sunyaev 1975, Sunyaev and Shakura 1977) from accreting onto the star.

\subsection{ADAF-DISK INTERACTION: LITHIUM}

One of the strong predictions of ADAF models, whether for black holes or neutron stars, is that the accreting plasma in the inner regions has an ion temperature of $10-100 \mathrm{MeV}$. Nearby is a cool and dense accretion disk feeding this plasma. If only a small fraction of the hot ion plasma gets in contact with the disk, the intense irradiation by ions will produce nuclear reactions (Aharonian and Sunyaev 1984, Martín et al. 1992). The main effects would be spallation of $\mathrm{CNO}$ elements into $\mathrm{Li}$ and $\mathrm{Be}$, and the release of neutrons by various reactions. In this context, it is intriguing that the secondaries of both neutron star and black hole accreters have high overabundances of Li compared with other stars of their spectral types (Martín et al. 1992, 1994a). If a fraction of the disk material is carried to the 
secondary by a disk wind, the observed Li abundances may be accounted for (Martín et al. 1994b).

\subsection{ADAF-DISK INTERACTION: HARD X-SPECTRA}

The interaction of a hot ion plasma with the cool disk produces a surface layer heated by the incident ions through Coulomb interactions with the electron gas in the disk. Its thickness and temperature turn out to be largely self-regulating: the energy balance is as in the Haardt and Maraschi corona models (in which the interaction is by photons only), while the optical thickness self-regulates through the dependence of the ion penetration depth on the electron temperature. This model (Spruit 1997) produces hard comptonized X-ray spectra whose shape is largely independent of both the energy flux and distance from the central object.

\section{Outflows?}

The energy density in an advection dominated accretion flow is of the same order as the gravitational binding energy density $G M / r$, since a significant fraction of that energy went into internal energy of the gas by viscous dissipation, and little of it got lost by radiation. The gas is therefore only marginally bound in the gravitational potential. This suggests that perhaps a part of the accreting gas can escape, producing an outflow or wind. In the case of the ion supported optically thin ADAFs, this wind would be thermally driven by the temperature of the ions, like an 'evaporation'

from the accretion torus. In the case of the radiation supported tori, which exist only at a luminosity near the Eddington value, but with much lower temperatures than the ion tori, winds driven by radiation pressure could exist.

The possibility of outflows is enhanced by the viscous energy transport through the disk. In the case of thin accretion disks (not quite appropriate in the present case, but sufficient to demonstrate the effect), the local rate of gravitational energy release $\left(\mathrm{erg} \mathrm{cm}^{-2} \mathrm{~s}^{-1}\right)$ is $W=\Sigma v_{r} \partial_{r}(G M / r)$. The local viscous dissipation rate is $(9 / 4) \nu \Sigma \Omega^{2}$. They are related by

$$
Q_{\mathrm{v}}=3\left[1-\left(\frac{r_{\mathrm{i}}}{r}\right)^{1 / 2}\right] W
$$

where $r_{\mathrm{i}}$ is the inner edge of the disk (see 'accretion disks' elsewhere in this volume). The viscous dissipation rate is less than the gravitational energy release for $r<(4 / 9) r_{\mathrm{i}}$, and larger outside this radius. Part of the gravitational energy released in the inner disk is transported outward by the viscous stresses, so that the energy deposited in the gas is up to three times larger than expected from a local energy balance argument. The 
temperatures in an ADAF would be correspondingly larger. Begelman and Blandford (1999) have appealed to this effect to argue that in an ADAF most of the accreting mass of a disk might be expelled through a wind, the energy needed for this being supplied by the viscous energy transport associated with the small amount of mass that actually accretes.

These suggestions are in principle testable, since the arguments are about two-dimensional time dependent flows (axisymmetric), which can be studied fairly well by numerical simulation. Igumenshchev et al. (1996), and Igumenshchev and Abramowicz (1999) present results of such simulations, but unfortunately these give a somewhat ambiguous answer to the question. For large viscosity $(\alpha \sim 0.3)$ no outflow is seen, but for small viscosity time dependent flows are seen with outflows in some regions. Some

of these flows may be a form of convection and unrelated to systematic outflows.

\section{References}

Abramowicz M.A., Czerny B., Lasota J.P., Szuszkiewicz E., 1988 Astrophys. J. 332, 646 Abramowicz M.A, Kato S., Matsumoto R., 1989, Publ. Astr. Soc. Japan 41, 1215

Aharonian F.A., Sunyaev, R.A., 1984 Mon. Not. R. astron. Soc. 210, 257

Begelman M.C., Meier D.L., 1982, Astrophys. J. 253, 873

Begelman M.C., Blandford R.D., 1999, Mon. Not. R. astron. Soc. 303, L1

Bisnovatyi-Kogan G.S., Lamzin S.A., 1984, Astron. Zh 61, 323 (translation Soviet Astron. 28, 187)

Bisnovatyi-Kogan G.S., Blinnikov S.I., 1976, Pi'sma Astron. Zh 2, 489 (translation 1977, Soviet Astron.L 2, 191)

Bisnovatyi-Kogan G.S., Blinnikov S.I., 1977, Astron. Astrophys. 59, 111

Cannon R.C., Eggleton P.P., Zytkow A.N., Podsiadlowski P., 1992, Astrophys. J. 386, 206

Chen X.M., Abramowicz M.A., Lasota J.-P., Narayan R., Yi I., 1995, Astrophys. J. 443, L61

Chevalier R.A., 1993, Astrophys. J. 411, 33

Di Matteo T., Celotti A., Fabian A.C., 1999a, Mon. Not. R. astron. Soc. 304, 809

Di Matteo T., Fabian A. C., Rees M.J., Carilli C.L., Ivison R.J.,1999b, Mon. Not. R. astron. Soc. 305, 492

Esin A.A., Narayan R., Cui W., Grove J.E., Zhang S.N., 1998, Astrophys. J. 505, 854

Frank, J., King, A.R., and Raine, D.J., 1992, Accretion Power in Astrophysics (2nd edition), Cambridge University Press.

Galeev A.A., Rosner R., Vaiana G.S., 1979, Astrophys. J. 229, 318

Gilham S., 1981, Mon. Not. R. astron. Soc. 195, 755

Haardt F., Maraschi L., 1993, Astrophys. J. 413, 507

Honma F., Kato S., Matsumoto R., Abramowicz M., 1991, Publ. Astr. Soc. Japan 43, 261

Ichimaru S., 1977, Astrophys. J. 241, 840

Igumenshchev I.V., Chen X.M., Abramowicz A., 1996 Mon. Not. R. astron. Soc. 278, 236

Igumenshchev I.V., Abramowicz M.A., 1999, Mon. Not. R. astron. Soc. 303, 309

Illarionov A.F., Sunyaev R.A., 1975, Astron. Astrophys. 39, 185

Kato M., 1997, Astrophys. J. Suppl. 113, 121

King, A.R., 1995 in Lewin, W.H.G., van Paradijs, J., van den Heuvel, E.P.J., eds., X-Ray 
Binaries, Cambridge University press, p.419

King A.R. and Ritter H., 1999 Mon. Not. R. astron. Soc. 309, 253

King A.R., Begelman M.C., 1999, Astrophys. J. 519, L169

Lee J.C., Fabian W.N., Brandt W.N., Reynolds C.S., Iwasawa K., 1999, astro-ph/9907352

Loeb A., Laor A., 1992, Astrophys. J. 384, 115

Lynden-Bell D., Pringle J.E., 1974, Mon. Not. R. astron. Soc. 168, 603

Martín E L., Rebolo R., Casares J., Charles P.A., 1992, Nature 358, 129

Martín E.L., Rebolo R., Casares J., Charles P.A., 1994a Astrophys. J. 435, 791

Martín E., Spruit H.C., van Paradijs J., 1994b, Astron. Astrophys. 291, L43

Meyer F., Meyer-Hofmeister E., 1994, Astron. Astrophys. 288, 175

Meyer-Hofmeister E., Meyer F., 1999, astro-ph/9906305

Nakamura K.E., Matsumoto R., Kusunose M., Kato S., 1996, Publ. Astr. Soc. Japan 48, 769

Narayan R., Yi I., 1994, Astrophys. J. 428, L13

Narayan R., Yi I., Mahadevan R., 1995, Nature 374, 623

Narayan R., Kato S., Honma F., 1997, Astrophys. J. 476, 49

Nayakshin S., Rappaport S., Melia F., 1999, astro-ph/9905371

Ogilvie G.I., 1999, Mon. Not. R. astron. Soc. 306, L9

Podolak M., Hubbard W.B. Pollack J.B., 1993, in Protostars and Planets II, University of Arizona Press, p.1109

Popham R., Woosley S.E., Fryer C., 1999, Astrophys. J. 518, 356

Quataert E., Narayan R., 1999 Astrophys. J. 517, 101

Rappaport S., Podsiadlowski P., 1999, astro-ph/9906045

Rees, M.J., 1978, Physica Scripta 17, 193

Rees M.J., Phinney E.S., Begelman M.C., Blandford R.D., 1982, Nature 295, 17

Sakimoto P., Coroniti F.V., 1989, Astrophys. J. 342, 49

Schultz A.L., Price R.H., 1985, Astrophys. J. 291, 1

Shapiro S.L., Lightman A.P., Eardley D.M., 1976 Astrophys. J. 204, 187

Sunyaev R.A., Shakura N.I., 1975, Pi'sma Astron. Zh. 3, 216 (translation Soviet Astron. L. 3, 114)

Spitzer L., 1965, Physics of fully ionized gases, Interscience Tracts on Physics and Astronomy, New York: Interscience Publication, 1965, 2nd rev.

Spruit H.C., Matsuda T., Inoue M., Sawada K., 1987, Mon. Not. R. astron. Soc. 229, 517

Spruit H.C., 1997, in Accretion disks-new aspects, eds. E. Meyer-Hofmeister and H.C. Spruit, Lecture Notes in Physics 487, Springer, p. 67

Taam R.E., 1994, in Compact stars in Binaries (IAU Symp 165), J. van Paradijs et al. eds., Kluwer, p.3

Taam R.E., 2000, ARAA, in prepapration

van Paradijs J., McClintock J.E., 1995, in Lewin, W.H.G., van Paradijs, J., van den Heuvel, E.P.J., eds., X-Ray Binaries, Cambridge University press, p.58

Wang J.M., Zhou Y.Y., 1999 Astrophys. J. 516, 420

Yi I., 1999, in Astrophysical Discs, eds. J.A. Sellwood and J. Goodman, Astronomical Society of the Pacific Conference series 160, 279. (astro-ph/9905215)

Zdziarski A.A., 1998, Mon. Not. R. astron. Soc. 296, L51

Zeldovich Ya.B., Ivanova J., Nadyozhin D.K., 1972. Soviet Astron. 16, 209 